\title{
La couleur rouge et sa valeur pathétique dans l'expressionnisme abstrait et le surréalisme tardif
}

\author{
The Color Red and its Pathetic Value in Abstract Expressionism \\ and Late Surrealism
}

\section{RÉSUMÉ}

On analyse dans cet essai le dialogue entre art visuel (l'automatisme de Jean-Paul Riopelle) et surréalisme tardif sur la couleur rouge, notamment en termes d'intensité. Le point de départ est un poème en prose d'André Breton, Élisa Breton, et Benjamin Péret, écrit pour une exposition de 1949 et intitulé « Riopelle ». Ce texte a été repris dans Le Surréalisme et la peinture.

Mots-clés : rouge, surréalisme, expresionnisme abstrait, Riopelle, Breton

\section{ABSTRACT}

This essay analyses the dialogue between automatism and late surrealism on the color red, with a focus on the intensity shared by painting and literature. The starting point is a collective prose poem by André Breton, Élisa Breton, and Benjamin Péret. This poem, "Riopelle », written for an exhibition of Jean-Paul Riopelle's automatist paintings in 1949, is included in Breton's Le Surréalisme et la peinture.

Keywords: red, Surrealism, Abstract Expressionism, Riopelle, Breton

[ ] l'aeil aussi a ses orgies et l'idée ses réjouissances.

Flaubert

\section{Introduction}

Flaubert écrivait à Louise Colet que « la poésie de l'avenir » serait « une manière de voir » [italiques de l'auteur cité] (Flaubert 1980, p. 229), et que dans ces conditions, la littérature serait « surtout exposante » [italiques de l'auteur cité] (Flaubert, 1980, p. 298), sans cependant tomber dans le didactisme. Dès lors, selon lui, « [il] faut faire des tableaux, montrer la nature telle qu'elle est, mais des tableaux complets, peindre le dessous et le dessus » (Flaubert, 1980, p. 298). Ce plaidoyer fait de l'écrivain une manière de peintre, mais le domaine de ce peintre serait à la fois le visible et l'invisible. Il semble en fait que pour cet 'œil littéraire', l'important ne

Christophe Ippolito, Georgia Institute of Technology, School of Modern Languages, 613 Cherry Street, Atlanta, GA 30332-0375, christophe.ippolito@modlangs.gatech.edu, 
soit pas ce qui est décrit, mais la manière de le voir (qui contribuerait à constituer un style), le regard et non pas la chose regardée. Ce regard-style se déclinerait en regard de texte (pour une œuvre considérée), voire en regard de fragment, et les deux derniers seraient les projections du premier. Ce regard joue sur ce qui lui est déjà familier, et même le déjà-vu implique un jugement fondé sur une culture du regard, qui établit des classements, et réveille les échos de la mémoire de l'auteur, de certains de ses textes, et des textes (ou des images) sur lesquels ces derniers s'appuient. Ainsi dans cette phrase :

De Hagios Joannis jusqu'ici (Polignia) c'est une charmante route, paysage classique s'il en fut, tranquille; on a vu cela dans d'anciennes gravures, dans des tableaux noirs qui étaient dans des angles, à la place la moins visible de l'appartement [italiques de l'auteur cité] (Flaubert, 1964, p. 675).

Et en retour, cette mémoire favorise l'exercice d'une contemplation esthétique. Mais qu'arrive-t-il quand cette contemplation se fait sur une œuvre d'art qui fait naître chez l'écrivain non pas un sentiment familier, éthéré, passif ou tranquille, mais, au-delà du déjà-vu, un sentiment intense provoqué par la vision de la matérialité d'une œuvre d'art? «Je me souviens d'avoir eu des battements de cœur, d'avoir ressenti un plaisir violent en contemplant un mur de l'Acropole, un mur tout nu [...]. Eh bien! je me demande si un livre, indépendamment de ce qu'il dit, ne peut pas produire le même effet » (Flaubert, 2007, p. 31 ; lettre du 3 avril 1876 à George Sand). Il s'agit là d'un ravissement que Huysmans ne trouve pas chez Gautier :

L'impression des objets s'était fixée sur son œil si perceptif, mais elle s’y était localisée, n'avait pas pénétré plus avant dans sa cervelle et dans sa chair; de même qu'un prodigieux réflecteur, il s'était constamment borné à réverbérer, avec une impersonnelle netteté, des alentours (Huysmans, 1977, p. 308).

L'intensité de cet effet-œuvre peut-elle être reproduite par un texte littéraire? Tel est le problème que pose cette phrase de Flaubert, tel est le nœud du texte qu'on va étudier, dans la mesure où précisément ce texte semble tenter de reproduire un effet similaire à celui des paysages ou monuments dont Flaubert parle. Dans ce texte surréaliste, si la manière de voir reste l'essentiel, la recherche de l'intensité sur laquelle jouent les co-auteurs semble, comme dans le cas de Flaubert, devoir prédominer. Et comme pour ce mur de l'Acropole, la beauté et la littérarité des éléments ci-après semblent partager un élément fondamental de surprise : on est à cent lieues du familier. Mais, comme chez Flaubert, on est encore dans le domaine de la littérature exposante, et dans la partie généralement considérée la plus visuelle de celle-ci : l'ekphrasis. 


\section{Le rouge en contexte : entre expressionnisme abstrait et surréalisme tardif}

Pour mieux comprendre les liens qui unissent littérature exposante et peinture, on voudrait analyser ici les modalités du transfert et du dialogue entre art visuel (l'automatisme de Jean-Paul Riopelle) et surréalisme tardif (André et Élisa Breton, et Benjamin Péret) sur le 'cri' de la couleur rouge. Le point de départ est un texte écrit pour une exposition de 1949 et intitulé Riopelle, un texte repris dans Le Surréalisme et la peinture (Breton, 1979, pp. 282-284) et qu'on ne peut reproduire ici dans son intégralité. Le lecteur est donc invité à s'y reporter. Il ne s'agit pas d'un texte sur un tableau précis de Riopelle, bien que son tableau La Ville soit reproduit dans le catalogue de l'exposition, catalogue introduit par ce texte ; c'est plutôt un texte sur l'œuvre de l'artiste en général et ce qu'elle tend à exprimer selon les trois co-auteurs, voire sur son entreprise et le sens de celle-ci. C'est là un type de critique d'art créatrice qui prend la forme d'un poème fait en collaboration. Dans ce poème, cette phrase de Breton illustre bien ce que les trois co-auteurs ont cherché à faire : « Pour moi, Riopelle est le nuage qui sert de parachute à la carcasse de fer d'un immeuble toujours en construction ». On trouvera ci-dessous une tentative d'explication à cette métaphore du nuage, mais pour le moment notons combien l'image de Breton est proche du célèbre tableau de Duchamp exposé au musée des beaux-arts de Philadelphie et également centré sur l'expression du désir, La Mariée mise à nu par ses célibataires, même. Cependant cette métaphore ne suffit pas à rendre compte de la 'description d'œuvre' et du 'regard de texte' des trois co-auteurs. Ce sont en fait les variations à propos de la couleur rouge, essentielle dans l'œuvre de Riopelle, qui sont au centre du texte. Elles suggèrent une vision moderniste du monde.

\subsection{Un rouge moderniste}

On s'intéressera donc ici au rouge moderniste dans sa version surréaliste, entre peinture et littérature. Mais comment ce rouge est-il introduit dans l'histoire du texte et dans l'espace du tableau, dans la logique de l'œuvre d'art? Les couleurs se mélangent, impliquent mobilité, effacement, surimpression, interaction, rencontre, opposition. La couleur est lieu d'une interaction entre les arts, mais si elle est parfois considérée comme l'essence de la peinture, elle apparaît aussi comme l'attribut d'une fonction souvent décriée de la littérature, la description, et ce en particulier au sein d'un surréalisme qui ne célèbre pas précisément la description. Cette introduction de la couleur, on l'étudiera d'abord dans le similidialogue que met en scène le poème collectif des poètes surréalistes précités, ce qui conduira à s'interroger sur l'intensité qui semble ici constitutive du rouge.

\subsection{Entre peinture et poésie}

Eluard, commentant un tableau de Picasso, écrit que « [le peintre] est devant un poème comme le poète devant un tableau » (Eluard, 1968, p. 938). Chez Breton, 
ce simili-chiasme n'est pas figé ; on peut noter une évolution de l'attitude du poète par rapport aux tableaux. Dans le domaine de la couleur (peu présente dans la première version de 1928 du Surréalisme et la peinture), c'est plutôt dans des textes tardifs, comme dans celui qu'on étudiera, que la vision de Breton s'exprime. Mais dans le poème considéré intervient aussi la passion originaire de Breton pour le dialogique. «C'est encore au dialogue que les formes du langage surréaliste s'appliquent le mieux ", lit-on dans les Manifestes du surréalisme (Breton, 1973, p. 48). Dans ce poème il y a dialogue entre Breton (qui a repris le mouvement surréaliste en main depuis qu'il a organisé l'Exposition Internationale du Surréalisme à la galerie Maeght en juin 1947), Péret et Élisa Breton d'une part, et l'œuvre d'un artiste, Riopelle, d'autre part. Chaque intervention, si elle semble être d'abord un monologue, s'ouvre aux autres, les reprend, comme mimétiquement le pinceau procède par touches.

\subsection{En territoire automatiste}

On sait que pendant la deuxième guerre mondiale, Breton passe au Canada, où il écrira Arcane 17. Quelques mots sur Riopelle (1923-2002) : c'est un peintre canadien, ami d'Émile Borduas, et membre du groupe des automatistes créé par Borduas dès 1946 ; ce groupe a recours aux principes de l'écriture automatique. Riopelle part à New York, à Paris, rencontre Mabille, Césaire, George Duthuit, et Georges Mathieu. Il se ralliera à l'abstraction lyrique puis à l'expressionnisme abstrait. Il a été le compagnon du peintre américain Joan Mitchell.

C'est la période automatiste de l'œuvre qui est examinée ici par les protagonistes à l'occasion de l'exposition personnelle de Riopelle à la galerie 'La Dragonne' en 1949. Rappelons la définition de l'automatisme, si essentiel à la conception que Breton a de l'art :

Je soutiens que l'automatisme graphique, aussi bien que verbal, sans préjudice des tensions individuelles profondes qu'il a le mérite de manifester et dans une certaine mesure de résoudre, est le seul mode d'expression qui satisfasse pleinement l'œil ou l'oreille en réalisant l'unité rythmique (aussi appréciable dans le dessin, le texte automatique que dans la mélodie ou dans le nid), la seule structure qui réponde à la non-distinction, de mieux en mieux établie, des qualités sensibles et des qualités formelles, à la non-distinction, de mieux en mieux établie, des fonctions sensitives et des fonctions intellectuelles (et c'est par là qu'il est seul à satisfaire également l'esprit). Que l'automatisme puisse entrer en composition, en peinture comme en poésie, avec certaines intentions préméditées, soit, mais on risque fort de sortir du surréalisme si l'automatisme cesse de cheminer au moins sous roche [italiques de l'auteur cité] (Breton, 1979, pp. 94-96).

Dans le poème considéré, il faudrait parler d'automatisme collectif. Cet « aparté » (le mot est mis en exergue du poème) est en fait conjonction de trois apartés par Breton, Péret et Élisa Breton ; les apartés se font écho, et ressemblent graphiquement à un dialogue constitué de répliques, même si la continuité 
apparente de ce dialogue se révèle largement artificielle lorsqu'on prend la peine d'en examiner les contenus. Le statut du texte est ambigu, entre critique d'art, poème-objet, et cadavre exquis. Rappelons que pour Breton le poème-objet « est une composition qui tend à combiner les ressources de la poésie et de la plastique et à spéculer sur leur pouvoir d'exaltation réciproque » (Breton, 1979, p. 365). Pour reprendre l'analyse de Michael Riffaterre, la critique d'art de Breton, " quintessentiellement littéraire », est en fait « une variante en prose de la poésie lyrique, l'expression du moi » (Riffaterre, 1991, p. 133), et se révèle ici comme un « discours du moi » qui se substitue à la critique. Et dans la mesure où le texte présente « avec des mots une représentation visuelle», il donne lieu à une ekphrasis, ou plus précisément à une « ekphrasis lyrique » (p. 133), qui tend par ailleurs à démentir la condamnation que fit Breton de la description. Lyrique car Breton et ses pairs racontent leurs émotions « sur le mode visionnaire » (p. 135).

\subsection{Points de comparaison}

La vision est manifestation d'une création. Le poème explore métaphoriquement les voies d'un 'orgasme' créatif représenté dans l'espace d'un tableau. Le « nuage » du poème considéré, parachute et orage en puissance, comme dans La Mariée de Duchamp, est ce qui contrôle la machine célibataire avant l'explosion. Le nuage est inscrit, comme souvent chez les surréalistes, dans une thématique romantique ; il est comme un lieu privilégié de la couleur dans la nature, un faisceau de couleurs en puissance ; le nuage a servi aux plus grands coloristes qui ont surenchéri sur sa mobilité, sa versatilité, sa capacité à générer de la couleur, bref, sur ce qu'on pourrait appeler sa capacité d' "imaginarisation », ainsi chez Bernardin de Saint-Pierre :

J'ai vu aussi dans les nuages des tropiques, de toutes les couleurs qu'on puisse apercevoir sur la terre, principalement sur la mer et dans les tempêtes. Il y en a alors de cuivrées, de couleur de fumée de pipe, de brunes, de rousses, de noires, de grises, de livides, de couleur marron, et de celle de gueule de four enflammé. Quant à celles qui y paraissent dans les jours sereins, il y en a de si vives et de si éclatantes qu'on n'en verra jamais de semblables dans aucun palais, quand on y rassemblerait toutes les pierreries du Mogol (Bernardin de Saint-Pierre, 2007, p. 17).

Le nuage est donc manifestation de couleur autant que possibilité de couleur, et ce qui domine dans ce texte de Bernardin, comme dans le poème considéré, est la couleur rouge (et ses différents tons). Cependant le poème du trio surréaliste commence par un blanc, le blanc du nuage, puis il y aura les blancs entre les apartés, et des couleurs non-vives : dans le texte, une carcasse de fer reprend les constructions de l'époque grise ou beige de Picasso (cf. Breton, 1979, p. 141). Dans le détail du poème, le « ça » qui se niche dans le syntagme « c'est » dévoile le pôle inconscient du refoulé, alors que les grues (animaux mais aussi machines, le texte joue sur le double sens) évoquent les machines d'un port comme les cris 
d'oiseaux en escale d'amour que Breton comme Riopelle avaient pu voir sur le Saint-Laurent. Les images qui suivent, avec la métaphore du trappeur, disent le désir et son élan. Or, comme on sait, le rouge est la couleur privilégiée pour dire le désir ou la passion; dans le contexte moderniste qui est celui de ce poème on retrouve cette couleur dans les références à un « port » et à une « mine» modernes. A propos de l'élan du désir, notons que dans « Pour se prendre au piège » in Capitale de la douleur, Eluard parle d'un orage qui permet de ne plus être le même (Eluard, 1966, p. 55). En ce sens, le texte fait partie d'un système poétique de références à d'autres textes qui constituent comme l'environnement sans lequel le poème ne peut véritablement se déchiffrer.

\subsection{Rouge désir}

Dans le poème, le désir se dit notamment par une association entre " port » et « mine » dans la réplique d'Élisa ; cette association est source d'un réseau connotatif qui implique un jeu sur les signifiants caractéristiques de ce poème en prose. Dans les images qui succèdent à cette 'association', une fête ouvre sur un prélude amoureux où l'homme est considéré plutôt comme machine désirante que comme être pensant, et où les sensations premières priment sur l'intellect. Dans la réplique de Breton (qui suit celle d'Élisa), les plis du vent miment ceux d'une robe mais aussi ceux de La Ville, tableau exposé par Riopelle en 1949 et représenté dans Le Surréalisme et la peinture avec le poème qui lui est contemporain. Puis, dans sa réplique (ou comme on voudra son aparté), Péret évoque une anecdote qui renvoie non pas aux abeilles de Virgile, mais à un conte du même Péret, Au paradis des Fantômes, où apparaît le personnage de Virgile de Naples, constructeur d'une mouche mécanique. Ce collage textuel est caractéristique d'un poème-objet.

Puis Élisa parle d'un lit qui dit la réalisation du désir, comme l'ouragan et les précipitations sont la réalisation de la potentialité 'nuage'; la force inhérente à la nature se libère (Pierre Schneider insiste dans une étude sur Riopelle sur ce phénomène ; Schneider, 1957-1958, pp. 85-87), et son précipité, à la fois dépôt sexuel et dépôt de couleur sur la toile, est aussi chute, tant il est vrai que « [toute] épave à la portée de nos mains doit être considérée comme un précipité de notre désir » (Breton, 1979, p. 363); ce précipité serait à la fois résultat de 1' 'orgasme' créatif évoqué plus haut et métaphore de la vitesse de l'automatisme. Dans la réplique de Breton qui suit, la paronomase dans le segment « Elle aime son ami et les lacs sombres » est une figure de l'union, alors que le sombre fait partie des valeurs picturales qui par contraste (et comme Klee et d'autres l'ont indiqué) 'contextualisent', 'installent' le rouge (voir sur ce point Victor Hugo, qui évoque dans Le Rhin « les sombres moires de la nuit», en un vers cité par Huguet, 1905, p. 249). 


\section{Vers un 'sur-rouge'}

Que penser de ce rouge surréaliste et automatiste à la fois ? Qui considère la réplique de Péret introduisant l'image de l'aurore boréale (phénomène à prédominante verte dans la nature, mais qui peut aussi être de couleur rougeâtre, rose ou violette) peut savoir que la flamme de l'aurore boréale est chez Breton l'emblème de l'amour ; c'est aussi une image de la création (et dans notre poème un commentaire sur l'utilisation du rouge dans les tableaux de Riopelle); on peut consulter sur ce point le Dictionnaire abrégé du surréalisme ; l'entrée « rouge » donne : « Récolte de la tomate par des cardinaux apoplectiques au bord de la mer Rouge (Effet d'aurore boréale) (Alphonse Allais) » (Breton \& Eluard, 1991, p. 75). Dans le poème étudié, Breton poursuit en évoquant l'état de grâce manifesté dans Arcane 17 ; les rosaces ne sont plus épanouies comme chez Gautier mais éclatent avec l'aboutissement du désir. Polysémie du rouge : le rouge de la flamme est création, forge, et intensité, festin, ivresse ; le rouge est couleur du feu, allié de la foudre, du ciel incendié, ensanglanté par l'aurore boréale et ses jambes de flammes ; il y a homophonie, homonymie de couleur dans deux arts différents. Le rouge colore ici une machine désirante à la Deleuze et Guattari. C'est la version moderne de la grande prostituée de l'apocalypse, c'est le rouge comme couleur de la passion, le rouge « amoureuse » (Eluard, 1966, p. 175). Et en ce sens l'utilisation du rouge reste compatible avec certaines valeurs traditionnelles associées à cette couleur, valeurs qu'illustrent les images de la vie et de la régénération présentes dans le sang et le feu, respectivement.

\subsection{Un rouge mimétique?}

Suit dans ce poème collectif une intervention brutale de Péret (" Tais-toi »), en contradiction totale avec le système théâtral de l'aparté ; Péret, vilipendeur de curés, prolonge l'image de la rosace éclatée avec celle du cadavre de curé (ici le rouge et le noir de la soutane sont en tension). Dans l'avant-dernière intervention d'Élisa qui suit, le fjord reprend l'image de l'aurore boréale. Chez Breton qui poursuit, les Indiens (en tant que 'Peaux-Rouges') prolongent la couleur dominante et le Nouveau Monde évoqué au début du texte, monde qui est l'univers du Canadien qu'est Riopelle; et les «fleuves [des] colliers » sont dans le texte une version surréaliste des rivières de diamants des bijoutiers. Dans les dernières interventions des trois poètes, la fin de «l'orage » est représentée par les emblèmes de l'automne ; et le biscuit de neige, état final du nuage, est aussi une sorte de précipité qui laisse son empreinte sur la toile. Avec ses rouges feux, l'automne, associé dans le poème aux fauves de la savane, à des feuilles d'automne très hugoliennes dans de grands bois où joue le soleil, ou encore à une amanite phalloïde (précipité rouge vif et coin de nature anti-naturelle dans le tableau peu à peu formé par le poème), est une 'expression abstraite' de l'été indien américain, selon certains commentateurs. Ainsi Waldberg (1981) a-t-il 
commenté une toile de Riopelle comme une « sorte de paysage rouge où dansent les couleurs d'une fête sauvage » (p. 77). Il reste que toute cette ekphrasis semble bâtie à partir de l'image matricielle du nuage comme métaphore du désir et du travail de la création. En devenant ouragan et / ou aurore boréale, ce nuage réalise ses potentialités, comme fait l'amour, comme fait l'œuvre.

\subsection{Lyrisme et 'surexpression'}

Il est cependant un autre rouge, moderniste lui aussi, qui brille ici par son absence : le rouge politique, emblème de la révolution, celui qu'on trouve par exemple dans l'Ode à Charles Fourier, texte contemporain à notre poème et où Breton se fait critique de son temps. Mais dans notre poème, le rouge sert plutôt à la description d'une image de la création soutenue par une vision lyrique. Précisons, comme l'a vu un commentateur, que des

[...] valeurs distinctes sont données par Breton et par les théoriciens cubistes et « post-cubistes » au terme de "lyrisme ». Chez Kahnweiler, le lyrisme fournit la résolution du conflit entre la représentation et la structure. Le lyrique, c'est la joie devant la beauté des choses, en dehors du narratif, du drame antique, de l'épopée, qui permet à la beauté formelle des choses de se marier avec la beauté formelle de la structure de la peinture (Ades, 1991, p. 36).

Comme l'a remarqué Combe, Breton a toujours cru en l'importance du sentiment et de l'émotion, le lyrisme étant pour lui « dépassement en quelque sorte psalmodique de l'expression contrôlée » (Breton, Entretiens, cité in Combe, 1991, p. 79). Et l'œuvre plastique se référera à ce modèle ou ne sera pas : «c'est bien l'exaspération du sentiment qui distingue le lyrisme de la surréalité de sa définition classique et le rattache au Romantisme [...] à travers le thème de la passion » (Combe, 1991, p. 81). Ainsi Breton parlera-t-il pour la peinture de Miró d'une peinture qui «ne demande au réel que le surexpressif, l'expressif au sens le plus enfantin », pour mieux distinguer l'expression de l'imitation, le lyrisme étant du côté de la création [italiques de l'auteur cité] (p. 83). Il revalorise ainsi l'inventio contre la dispositio (p. 89). Et « [1'] idée d'automatisme est en soi le recours le plus radical contre l'organisation du discours et de la peinture, et permet de se libérer de la tyrannie de la composition » (p. 89). Il est cependant vrai que comme chez Paulhan, « le refus de la rhétorique est encore une rhétorique (p. 93).

Au-delà, il semble qu'ici par l'intensité du lyrisme Riopelle saisisse « l'esprit de la plénitude de la nature », par l'intermédiaire d'un «élan d'activité » (Auster, 1981, p. 82), soit un phénomène qu'on peut relier sur le plan de la représentation élaborée dans notre poème à l'intensité de la couleur rouge, à l'affirmation du rouge. Du début à la fin du poème, il y a intensification des couleurs, du gris au noir, du violet au rouge. Le rouge est couleur par excellence, colorata; et en ce sens aussi, le rouge, extrême de la couleur, couleur per se, est intensité. Le rouge est énergie, couleur de la vivacité et de l'intensité, comme dans certains portraits 
d'Otto Dix, comme dans nombre de tableaux emblématiques de Bacon. Riopelle aime selon Hess (1981) les tons vifs, le rouge écarlate du tube, la difficulté à utiliser des couleurs qui « se comportent presque comme des teintures» (p. 83), et il aime à jouer sur la difficulté à les placer entre des tons plus neutres; or toujours selon Hess ces couleurs intenses ne peuvent jouer le rôle de couleurs que si elles sont « lourdement appliquées », " [généreusement] utilisées en taches, grumeaux, écumes » (p. 83) ; ainsi seulement elles ont une qualité pénétrante, comme des « joyaux » (p. 83).

\subsection{Energie et intensité de la couleur}

Dans ces conditions, observe Dupin (1981), « [1'] énergie de la couleur triomphe de l'inertie de la matière et de la dispersion des images » (p. 80). Vers le milieu des années 1950, Riopelle commence à peindre avec un couteau. A propos du résultat (« conglomérats denses de taches colorées » selon Schmalenbach, 1981, p. 78), et du vitalisme que ces taches expriment, Rusoli parle d' « amoncellements, pissements, éclats et traînées de couleur ». Selon sa formule, « [ 1]'épaisseur de la matière devient lumière. Et la lumière, substance, corps, se condense en des volumes différents » (Rusoli, 1981, p. 81). Comme si l'intensité s'accentuait encore. Schneider cite Riopelle à propos du rôle essentiel qu'il attribue à l'intensité :

On dit que la tache faite par une bouteille lancée du troisième étage est libre. Pas du tout. Le geste propre à chaque homme y est toujours. Sans parler du choix de la bouteille, de la couleur l'art, quoi. L'écriture automatique, c'est un climat intellectuel. Le véritable automatisme se situerait en dehors de l'individu. Où il y a geste, celui-ci est toujours guidé par l'individu. Il n'y a pas d'automatisme. Etre libre, c'est une autre façon d'être coincé, plus personnelle, plus intense. L'essentiel, c'est l'intensité (Riopelle, cité in Schneider, 1981, p. 15).

C'est sur ce point précis que Riopelle est le plus proche de Breton, pour qui

[1']émotion véhiculée par le lyrisme se caractérise par son intensité, ce que signifie encore la célèbre définition de la " beauté convulsive " dans L'Amour fou, " explosante-fixe ", et l'expression même d' «élan lyrique » utilisée à propos du cubisme (Combe, 1991, p. 81).

\subsection{De la valeur pathétique des couleurs}

On pourrait faire du texte des trois surréalistes un point de départ pour une interprétation phénoménologique de la couleur à même d'éclairer la création poétique, en insistant sur l'importance de l'intensité de la couleur du point de vue poïétique. On peut trouver une telle interprétation chez Cohen (1979), dans sa théorie phénoménologique de la poéticité. Ainsi Cohen écrit-il à propos du fameux syntagme «blanche agonie » de Mallarmé que dans le poème dont il est tiré « la blancheur [...] demeure seule dans l'univers de la couleur, comme si [...] toute agonie était blanche et toute blancheur mortelle» (p. 84). Le blanc, ou ici le rouge. Dès lors, la couleur peut avoir un sens « poétique, affectif ou pathétique » 
(p. 158). Dans ces conditions, le « pathème » que serait fondamentalement la couleur servirait à " désigner le contenu éprouvé de la signification » (p. 158), et aurait une intensité qu'on pourrait opposer aux autres instruments plus neutres de la représentation. Et en ce sens, on pourrait parler de " valeurs pathétiques » des couleurs, le rouge étant ainsi associé à la violence (p. 159 ; pour plus de précisions sur la théorie de Cohen, voir Ippolito, 2009, pp. 105-118).

\section{Conclusion}

On a examiné dans le poème considéré le sens qu'on peut donner à la dissémination métaphorique de la couleur rouge, aux réseaux culturels et thématiques qu'elle forme, à la polyphonie poétique engendrée par les diverses associations qui sont construites par les usages qui sont faits de cette couleur. On a comparé les rouges si singuliers qu'on y trouve à ceux utilisés par les grands coloristes en littérature, de Bernardin à Eluard. Mais il semble que dans le texte étudié, l'essentiel reste que le discours poétique soit ramené à sa fonction première, qui est de raconter des origines - théogonie, naissance d'un monde, d'une couleur, sorties de l'Idée au sein du langage. A proprement parler, il n'y a pas de critique de tableaux, mais plutôt une vision qui se libère de la toile pour devenir texte et raconter par un discours poétique l'aventure de la création : aventure signifiée par le processus métaphorique et l'engendrement des couleurs. Le rouge, figure de l'intensité, aiguille et gouverne ce processus, et ce dans une logique du et des sens qui n'a plus grand-chose à voir avec la représentation mimétique. Certes, « on peint mal, si une sorte de couleur universelle, particulière au sujet, ne domine pas constamment dans le tableau » (Senancour, 1984, p. 506), voire dans un texte comme celui-ci, anhistorique, tout entier orienté par le rapport à l'image (et la métaphore filée) de la création.

Au final, ce qui importe c'est que le regard transcende la description et par là la justifie poétiquement. Et par ailleurs, en termes d'opérations scripturales, Breton et ses co-auteurs se réapproprient certains instruments flaubertiens. L'écriture de la contemplation (que celle-ci soit désintéressée ou extatique), qui transparaît par exemple dans les silences ou les blancs de Flaubert, est utilisée dans notre poème pour donner un surplus de littérarité au décrit. Et les échos qui scandent les réseaux descriptifs qu'y forment les métaphores filées et autres sous-textes tissent, dans l'esprit de la prophétie flaubertienne sur la littérature exposante qu'on a citée au début de cet essai, une mémoire des 'dessous' du texte. 


\section{References}

Ades, D. (1991). Visions de la matière : Breton, Cubisme et Surréalisme. Pleine Marge, 13, 23-37.

Auster, P. (1981). Note. In M. Martin (Ed.), Jean-Paul Riopelle, Peinture 1946-1977 (p. 82). Paris: Centre Georges Pompidou.

Bernardin de Saint-Pierre, J.-H. (2007). Études de la nature. Saint-Étienne: Publications de l'Université de Saint-Étienne.

Breton, A. (1973). Manifestes du surréalisme. Paris: Gallimard/Jean-Jacques Pauvert.

Breton, A. (1979). Le Surréalisme et la peinture. Paris: Gallimard.

Breton, A., \& Eluard, P. (1991). Dictionnaire abrégé du surréalisme. Paris: José Corti.

Cohen, J. (1979). Le Haut Langage: théorie de la poéticité. Paris: Flammarion.

Combe, D. (1991). Rhétorique de la peinture. Pleine Marge, 13, 79-97.

De Senancour Pivert, É. (1984). Du style dans les descriptions. In J.-M. Monnoyer (Ed.), É. Pivert de Senancour. Obermann (pp. 503-513). Paris: Gallimard.

Dupin, J. (1981). Note. In M. Martin (Ed.), Jean-Paul Riopelle, Peinture 1946-1977 (p. 80). Paris: Centre Georges Pompidou.

Eluard, P. (1966). Capitale de la douleur. Paris: Gallimard.

Eluard, P. (1968). Euvres complètes: Vol. 2. Paris: Gallimard.

Flaubert, G. (1964). Voyage en Orient. In B. Masson (Ed.), Euvres complètes : Vol. 2. «L'Intégrale». Paris: Éditions du Seuil.

Flaubert, G. (1980). Correspondance: Vol. 2. Paris: Gallimard.

Flaubert, G. (2007). Correspondance : Vol. 5. Paris: Gallimard.

Hess, T. (1981). Note. In M. Martin (Ed.), Jean-Paul Riopelle, Peinture 1946-1977 (p. 83). Paris: Centre Georges Pompidou.

Huguet, E. (1905). La couleur, la lumière et l'ombre dans les métaphores de Victor Hugo. Paris: Hachette.

Huysmans, J.-K. (1977). À Rebours. Paris: Gallimard.

Ippolito, C. (2009). Le Chant du signifié : sur une lecture de Jean Cohen par Michel Houellebecq. L'Esprit Créateur, 49(2), 105-118.

Riffaterre, M. (1991). Ekphrasis lyrique. Pleine Marge, 13, 133-149.

Rusoli, F. (1981). Note. In: M. Martin (Ed.), Jean-Paul Riopelle, Peinture 1946-1977 (p. 81). Paris: Centre Georges Pompidou.

Schmalenbach, W. (1981). Note. In M. Martin (Ed.), Jean-Paul Riopelle, Peinture 1946-1977 (p. 78). Paris: Centre Georges Pompidou.

Schneider, P. (1957-1958). Jean-Paul Riopelle. Yale French Studies, 19/20, 85-93.

Schneider, P. (1981). Préface. In M. Martin (Ed.), Jean-Paul Riopelle, Peinture 1946-1977 (pp. 1116). Paris: Centre Georges Pompidou.

Waldberg, P. (1981). Note. In M. Martin (Ed.), Jean-Paul Riopelle, Peinture 1946-1977 (p. 77). Paris: Centre Georges Pompidou. 